\title{
Transient Hepatic Intensity Differences: Part 2, Those Not Associated with Focal Lesions
}

\section{Stefano Colagrande ${ }^{1}$ \\ Nicoletta Centi ${ }^{1}$ \\ Roberta Galdiero ${ }^{2}$ \\ Alfonso Ragozzino ${ }^{2}$}

Keywords: arterial phenomena, dynamic MRI, hemodynamics, liver, liver disease, liver perfusion abnormalities, MRI, THID, transient hepatic intensity differences

\section{DOI:10.2214/AJR.05.1367}

Received August 8, 2005; accepted after revision October 21, 2005.

1Department of Clinical Physiopathology, Section of Radiodiagnostics, University of Florence, Viale Morgagni 85, Florence 50134, Italy. Address correspondence to S. Colagrande (stefano.colagrande@unifi.it)

${ }^{2}$ Section of Radiodiagnostics, Ospedale SM Grazie Pozzuoli, Naples, Italy.

\section{AJR 2007; 188:160-166}

0361-803X/07/1881-160

(C) American Roentgen Ray Society

OBJECTIVE. The purpose of our study was to evaluate and to show the range of appearance of transient hepatic signal intensity differences when not associated with focal lesions, and to correlate morphology, cause, and pathogenesis.

CONCLUSION. Hepatic arterial phenomena, visualized on MRI and CT, must be considered important signs of underlying liver disorders, which these phenomena contribute to evaluate. Accordingly, the hepatic arterial phase must always be performed on MRI as well as on CT, even if no focal lesion is expected.

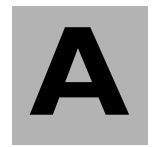
$\mathrm{s}$ stated in part 1 of this article [1], transient hepatic signal intensity differences can also not be associated with focal lesions. The aim of this second part of our article is to show their wide range of appearance and to rule out a possible diagnostic value. Transient hepatic intensity difference, in this case, can be induced by three pathogenetic mechanisms: portal hypoperfusion due to portal branch compression or thrombosis, flow diversion caused by arterioportal shunt or by an anomalous blood supply, and inflammation of the biliary vessels or adjacent organs [2, 3].

Transient hepatic intensity differences without a focal lesion may have three appearances: sectorial, which is triangular areas with a straight border that follows portal dichotomy; polymorphous, having various shapes and extension, usually without a straight border that does not follow portal dichotomy; and diffuse, having a varied appearance, which is the generalized equivalent of the sectorial transient hepatic intensity difference.

Accordingly, we have organized our article into three groups.

\section{Sectorial Differences}

This type of transient hepatic intensity difference is usually caused by portal hypoperfusion due to portal (Fig. 1) or hepatic vein thrombosis, long-standing biliary obstruction, or an arterioportal shunt, which may be congenital (Fig. 2) or, more frequently, due to liver cirrhosis or trauma [2, 4, 5] (Fig. 3). In an arterioportal shunt, mixing of venous lowpressure and arterial high-pressure blood leads to an arterial reaction with a similar mechanism to that of an aberrant venous supply or drainage or of shunts produced by a focal lesion (see part 1 [1]).

Sometimes transient hepatic intensity differences without a focal lesion may appear round because of a blockade in a portal or

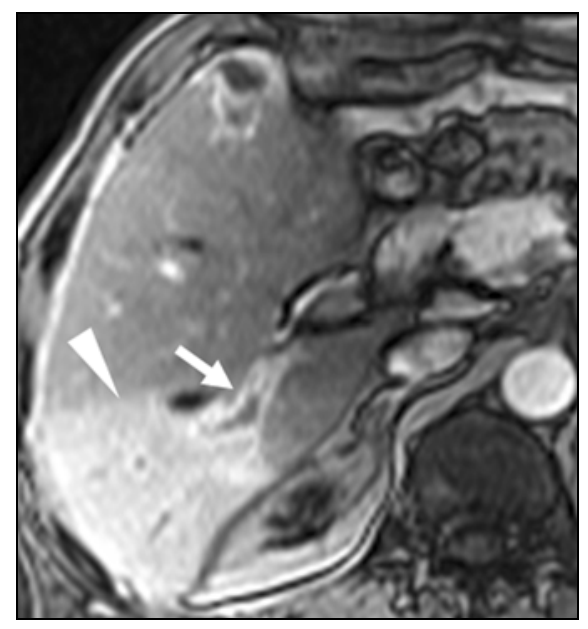

Fig. 1-58-year-old man with liver cirrhosis and sectorial transient hepatic intensity differences due to portal thrombosis secondary to hepatocellular carcinoma radiofrequency ablation. Axial gradientecho T1-weighted gadolinium-enhanced arterial phase image (TR/TE, 146/2) shows wedge-shaped arterial phenomenon (arrowhead) caused by portal branch thrombosis (arrow). Note also another hypointense treated nodule in anterior portion of liver segment $V$, with peripheral enhanced area due to arterial reaction. 


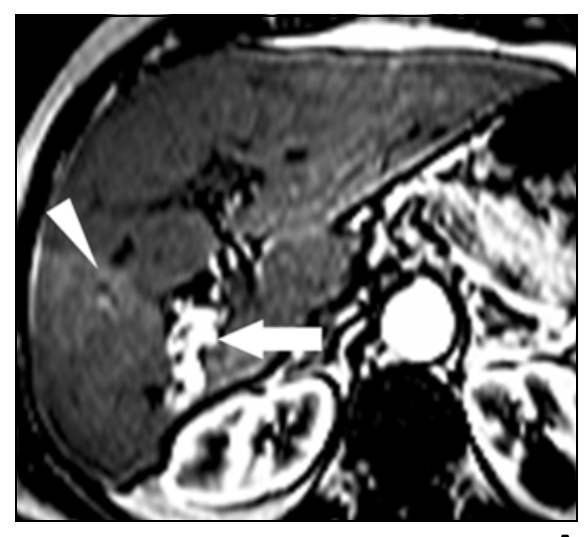

A

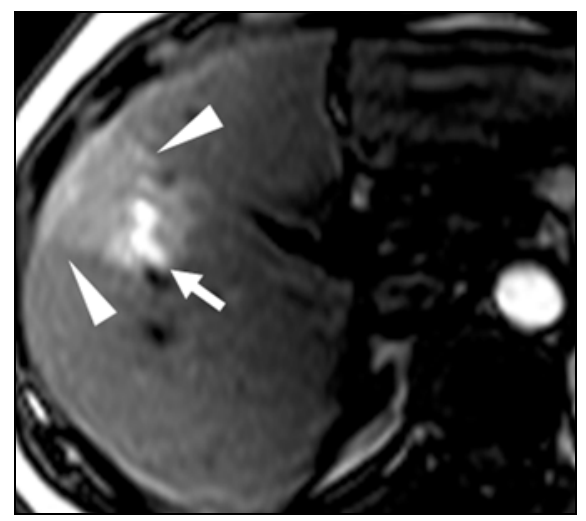

Fig. 3-42-year-old woman with sectorial transient hepatic intensity differences in right hepatic lobe caused by posttraumatic arterioportal shunt. Axial gradient-echo T1-weighted gadolinium-enhanced arterial phase MR image (TR/TE, 146/2) shows wedgeshaped arterial phenomenon (arrowheads) caused by arterioportal shunt (arrow) due to percutaneous hepatic biopsy performed 1 month earlier.

hepatic vein that does not reach the hepatic capsule (blind vessel). Moreover, sectorial transient hepatic intensity differences, especially when next to a Glisson's capsule, can show a pseudoglobular shape [2] that is due to the relationship between the position of arterialization in the liver and the MRI slice direction and orientation.

Pseudoglobular arterializations in the cirrhotic liver can create several differential diagnostic problems in hepatocellular carcinoma. However, unlike arterializations, hepatocellular carcinomas greater than 15 $\mathrm{mm}$ present $\mathrm{T} 1$ and $\mathrm{T} 2$ signal alterations on baseline scanning, show a washout phenomenon in the portal phase, and do not take up the hepatobiliary contrast agent on latephase MRI [6]. Nevertheless, diagnostic

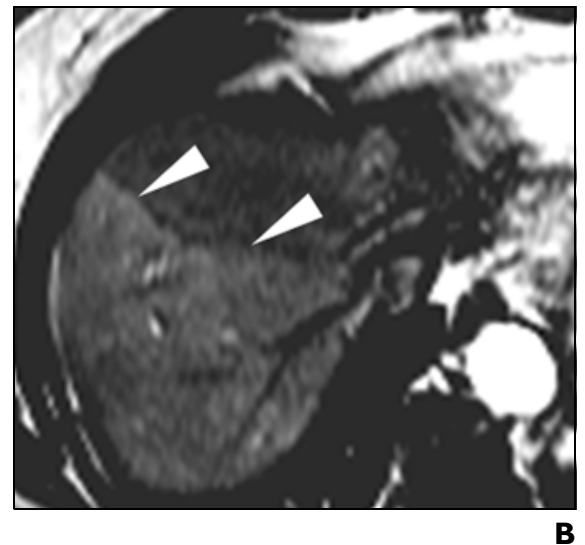

problems may persist in small, highly differentiated hepatocellular carcinomas (T1 and T2 isointensity, no washout, late concentration of hepatobiliary contrast agent).

In cases of cholangitis, sectorial transient hepatic intensity differences (Fig. 4) may be caused by peribiliary plexus impairment that leads to a decrease in portal blood flow to the sinusoids and arterial compensation. Moreover, arterializations caused by cholangitis may also assume other patterns (nodular, lobar, or diffuse) as a result of the wide range of presentation and extension of cholangitis [7] (Fig. 5).

\section{Polymorphous Differences}

Polymorphous arterial phenomena have four main causes.

The first cause is extrinsic compression of the liver surface exerted by ribs or stretched diaphragmatic pillars during deep inspiration or by subcapsular collections (Fig. 6). Such compression causes an increase of tissue pressure in the subcapsular region, which results in decreased portal perfusion and then in a usually slight arterial reaction [8].

Second, an anomalous blood supply may result from atypical arteries [9], collateral venous vessels, or accessory veins [8] (Fig. 7). The latter may act, accordingly to the pressure gradient, as anomalous supply or drainage vessels and are known as the "third hepatic inflow," which supplies small areas of the liver parenchyma, especially segment IV. These anomalous accessory veins enter the liver separately from the portal venous system. Nevertheless, they communicate with the intrahepatic portal branches to various extents, causing localized portal hypoperfusion and hepatic arterial perfusion reaction. These hemodynamic changes, if persistent, can also determine focal meta-
Fig. 2-63-year-old man with sectorial transient hepatic intensity difference in right hepatic lobe caused by congenital arterioportal shunt. A and B, Axial gradient-echo T1-weighted gadoliniumenhanced arterial phase MR images (TR/TE, 146/2) show arterial phenomenon (arrowheads) caused by arterioportal shunt (arrow, A).

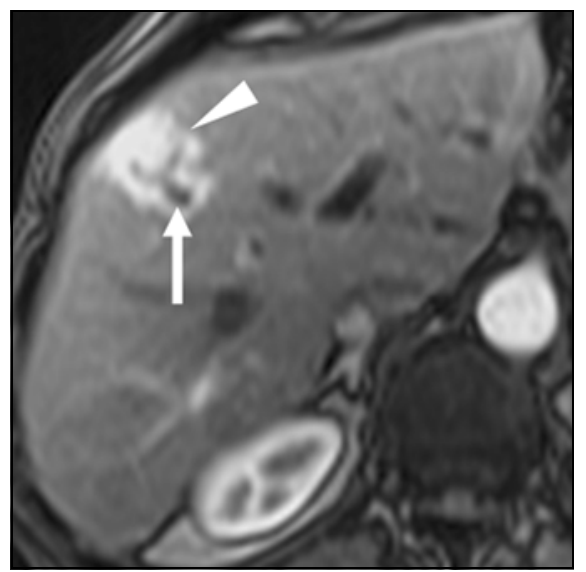

Fig. 4-61-year-old man with postbiopsy focal cholangitis and sectorial transient hepatic intensity differences. Axial gradient-echo T1-weighted gadolinium-enhanced arterial phase MR image (TR/TE, 146/2) shows wedgeshaped arterial phenomenon (arrowhead) caused by peribiliary plexus impairment secondary to dilation of inflamed subsegmental biliary vessels (arrow).

bolic alterations that result in focal sparing in fatty liver or nodular fat accumulation in the normal liver [2,8] (Fig. 8).

Third, in cases of inflammation of adjacent organs (cholecystitis, pancreatic abscesses) (Fig. 9), the morphogenesis of transient hepatic intensity differences is related to the spread of inflammatory mediators by contiguity. Increased arterial flow can also be secondary to portal inflow reduction due to interstitial edema [9].

Percutaneous hepatic biopsy and the outcome of interventional treatment of liver neoplasms, by physical or chemical agents, are the fourth cause of polymorphous transient hepatic intensity differences, whose peculiarity is their variety: 


\section{Colagrande et al.}

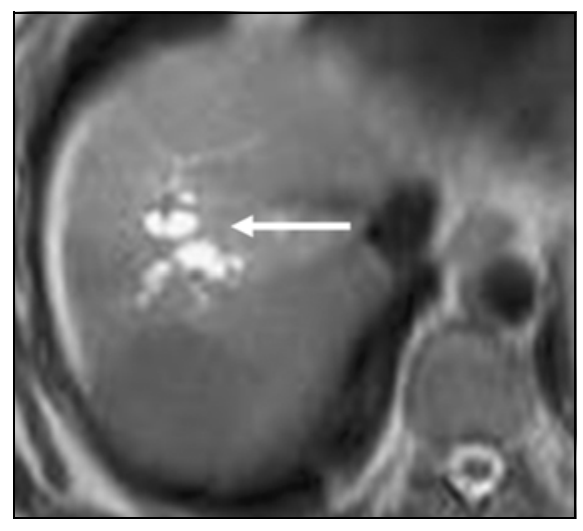

A

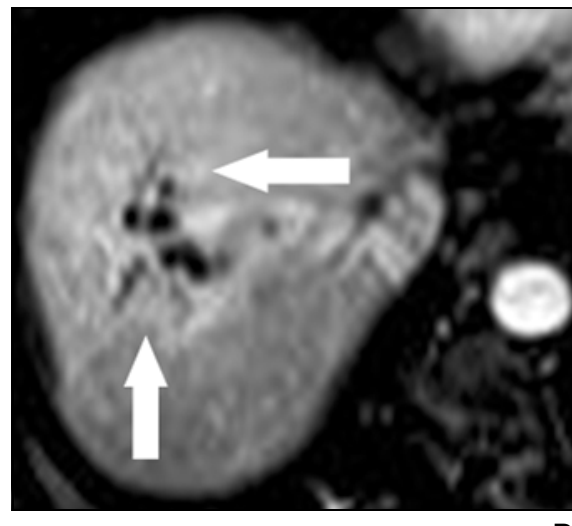

B

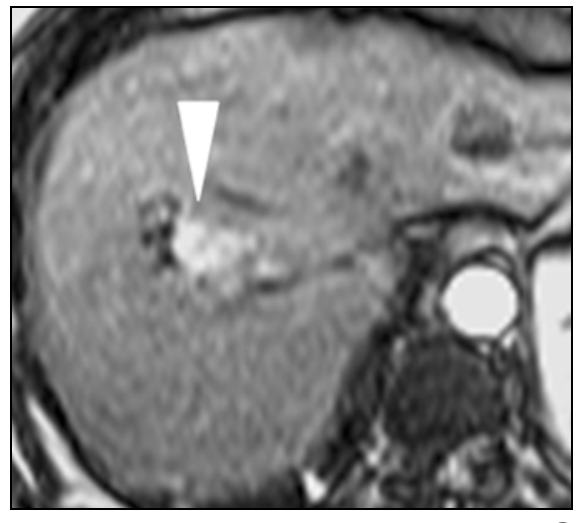

C

Fig. 5-57-year-old woman with cholangitis and nonsectorial transient hepatic intensity differences in hepatic dome.

A, Axial T2-weighted MR image (TR/TE, 12,000/82) shows localized dilation of bile ducts (arrow). Note small perihepatic effusion.

B and C, Axial gradient-echo T1-weighted gadolinium-enhanced arterial phase MR images (146/2) show further appearance of arterializations with biliary vessel disease: peribiliary (arrows, B), distributed along dilated biliary vessels, and pseudoglobular, mimicking a focal lesion (arrowhead, C).

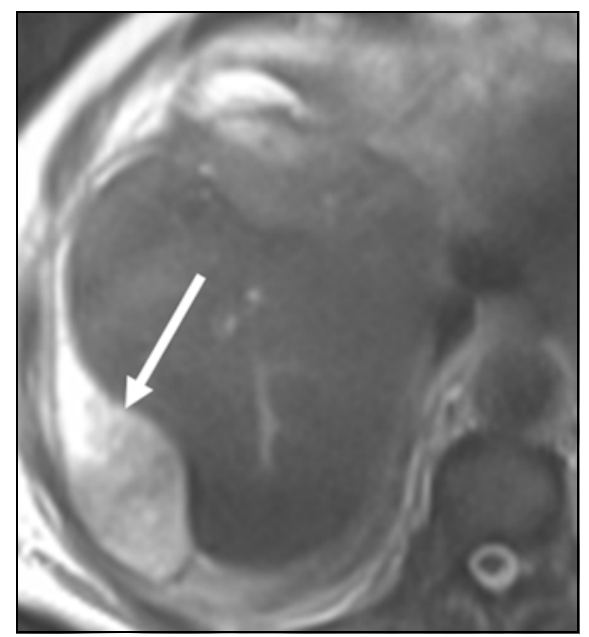

A
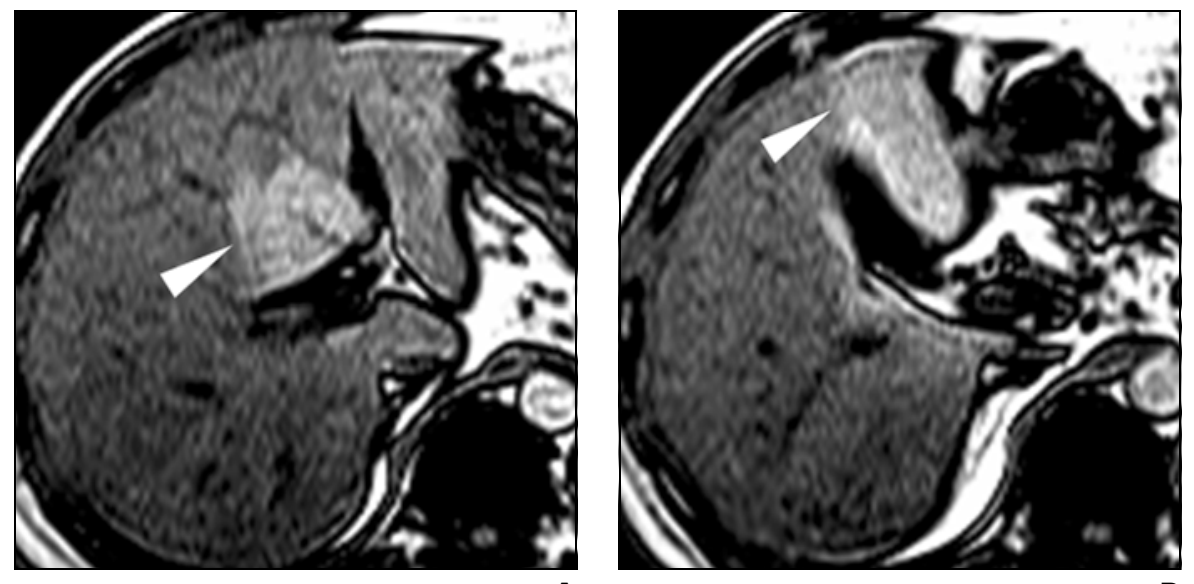

Fig. 6-69-year-old man with postsurgical subcapsular collection and polymorphous transient hepatic intensity differences.

A, Axial T2-weighted MR image (TR/TE, 12,000/84) reveals hyperintense collection (arrow) beneath Glisson's capsule, causing compression on adjacent marginal liver parenchyma.

B, Axial gradient-echo T1-weighted gadoliniumenhanced arterial phase MR image (146/2) shows polymorphous arterial phenomenon (arrowheads) laterally positioned to hypointense collection and caused by extrinsic compression of liver surface.

Fig. 7-40-year-old man with polymorphous transient hepatic intensity differences due to anomalous venous supply and drainage by right gastric vein.

A and B, Axial gradient-echo T1-weighted gadoliniumenhanced arterial phase MR images (TR/TE, 146/2) show polygonal arterial phenomenon in segment IV (arrowheads). 


\section{Transient Hepatic Intensity Differences}

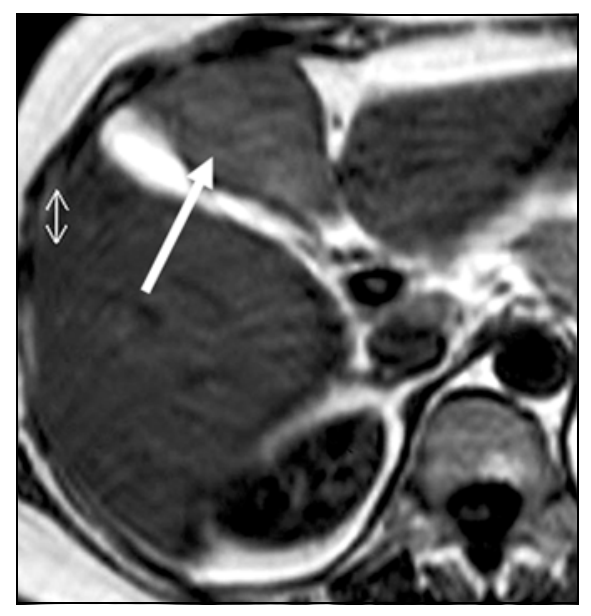

A

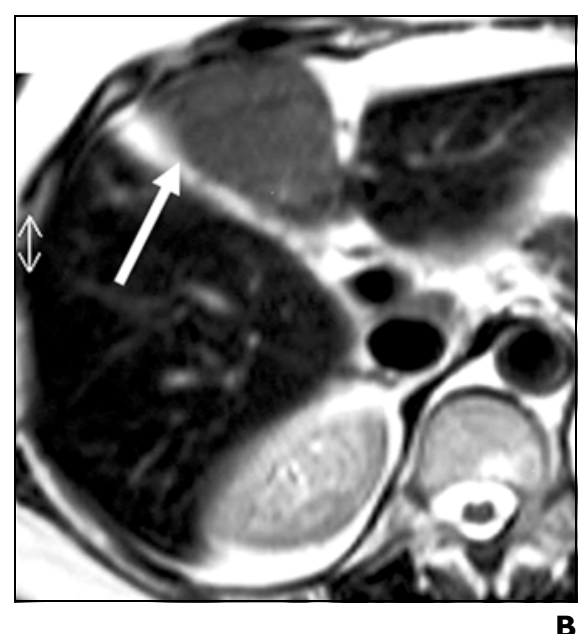

B

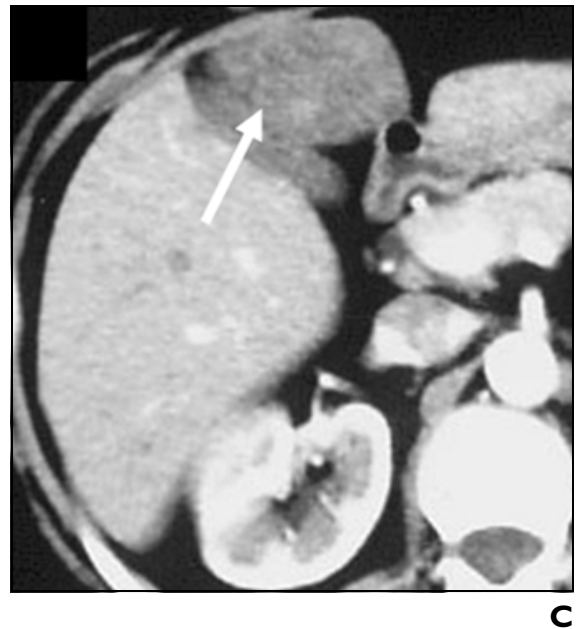

Fig. 8-56-year-old woman with segmental localized steatosis in liver segment IV due to persistent polymorphous perfusion alterations.

A and B, Axial gradient-echo T1-weighted (TR/TE, 216/1.5) (A) and axial T2-weighted (872/210) (B) MR images show signal intensity variation of segment IV (arrows) with respect to surrounding liver parenchyma.

C, Axial iodinated contrast-enhanced arterial phase helical CT image better shows relative hypodensity in segment IV (arrow) caused by segmental fat accumulation secondary to persistent hemodynamic changes.

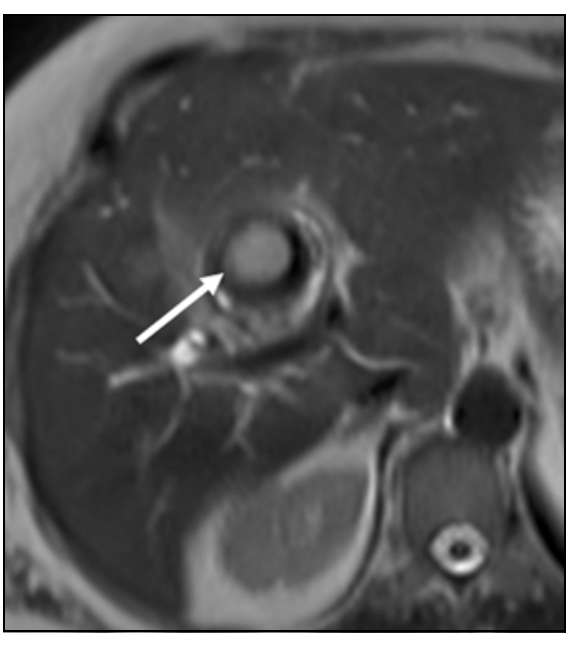

A

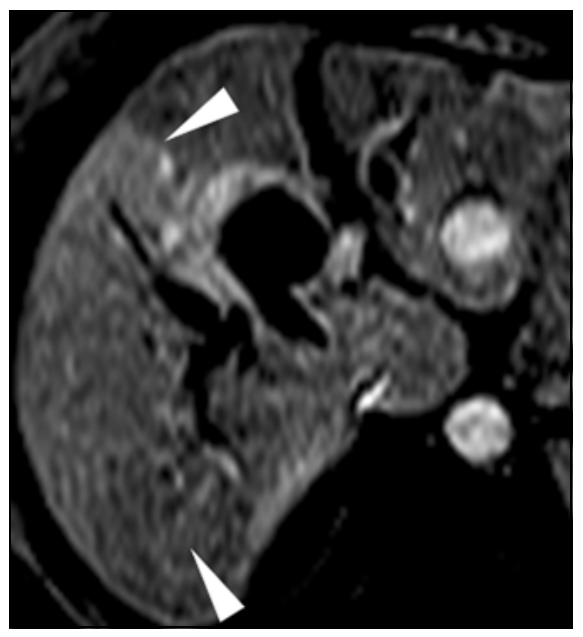

B
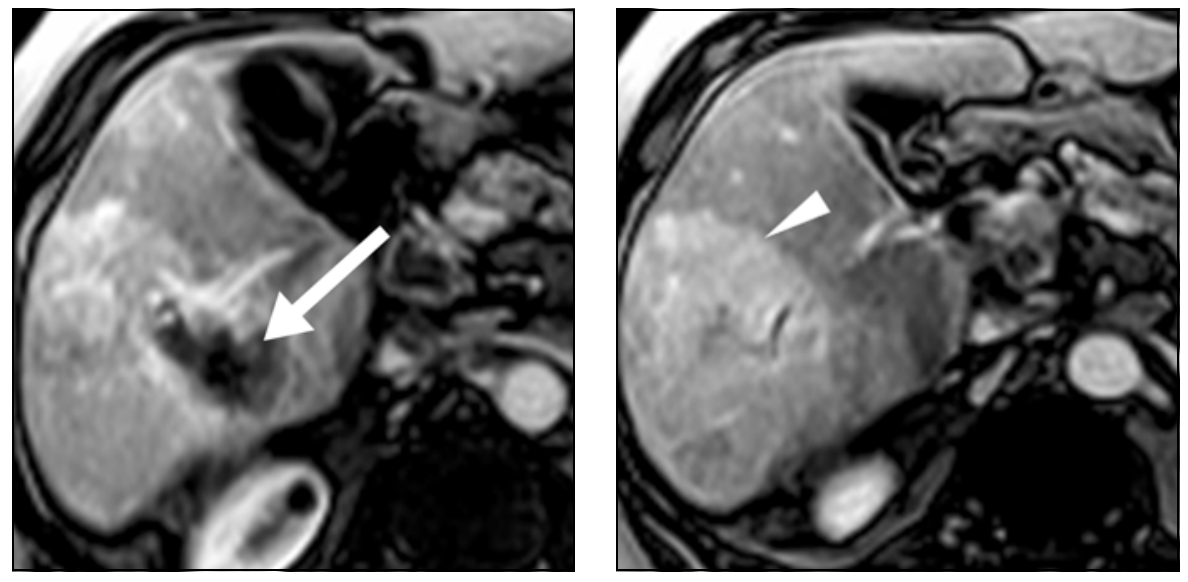

A

B
Fig. 9-63-year-old woman with gallstone causing inflammatory changes, main biliary duct compression (Mirizzi syndrome), and related polymorphous transient hepatic intensity differences.

A, Axial T2-weighted MR image (TR/TE, 12,000/84) reveals large calculus lodged in gallbladder (arrow), wall thickening, and slight parenchymal hyperintensity due to inflammation.

B, Axial gradient-echo T1-weighted gadoliniumenhanced arterial phase MR image (146/2) shows wide arterial phenomenon caused by spread of inflammatory mediators (arrowheads).

Fig. 10-60-year-old woman with previous hepatocellular carcinoma treated with radiofrequency ablation and polymorphous transient intensity difference in right hepatic lobe.

A and B, Axial gradient-echo T1-weighted gadoliniumenhanced arterial phase MR images (TR/TE, 146/2)

show irregularly shaped arterial phenomenon (arrowhead, B) positioned laterally to large hypointense lesion that is outcome of radiofrequency ablation (arrow). 


\section{Colagrande et al.}

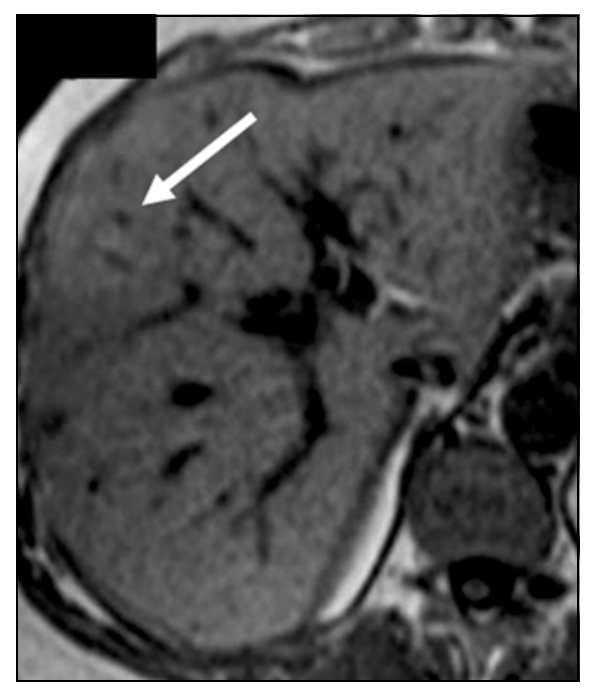

A

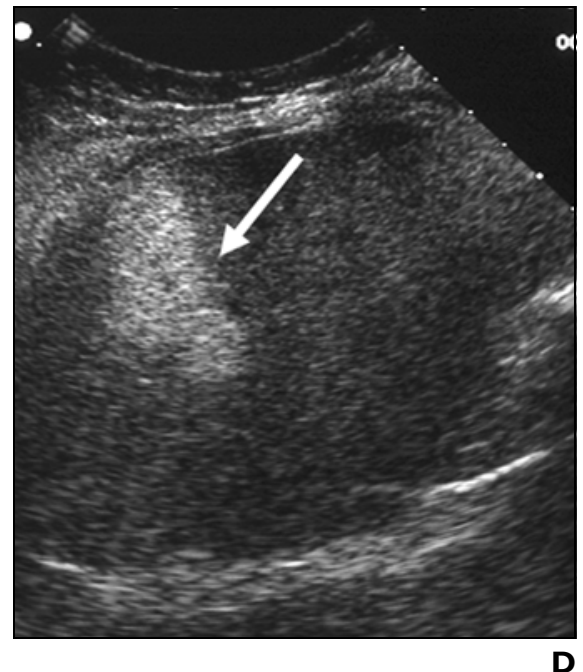

Fig. 12-23-year-old man with hepatic vein obstruction (Budd-Chiari syndrome) associated with a diffuse patchy pattern of transient hepatic intensity differences. Axial gradient-echo T1-weighted gadolinium-enhanced arterial phase MR image (TR/TE, 216/1.5) reveals diffuse marble aspect of liver parenchyma caused by sinusoidal obstruction and consequent transsinusoidal plexus activation. Hypointense oval structure in caudate lobe is due to obstructed transjugular intrahepatic portosystemic stent-shunt.
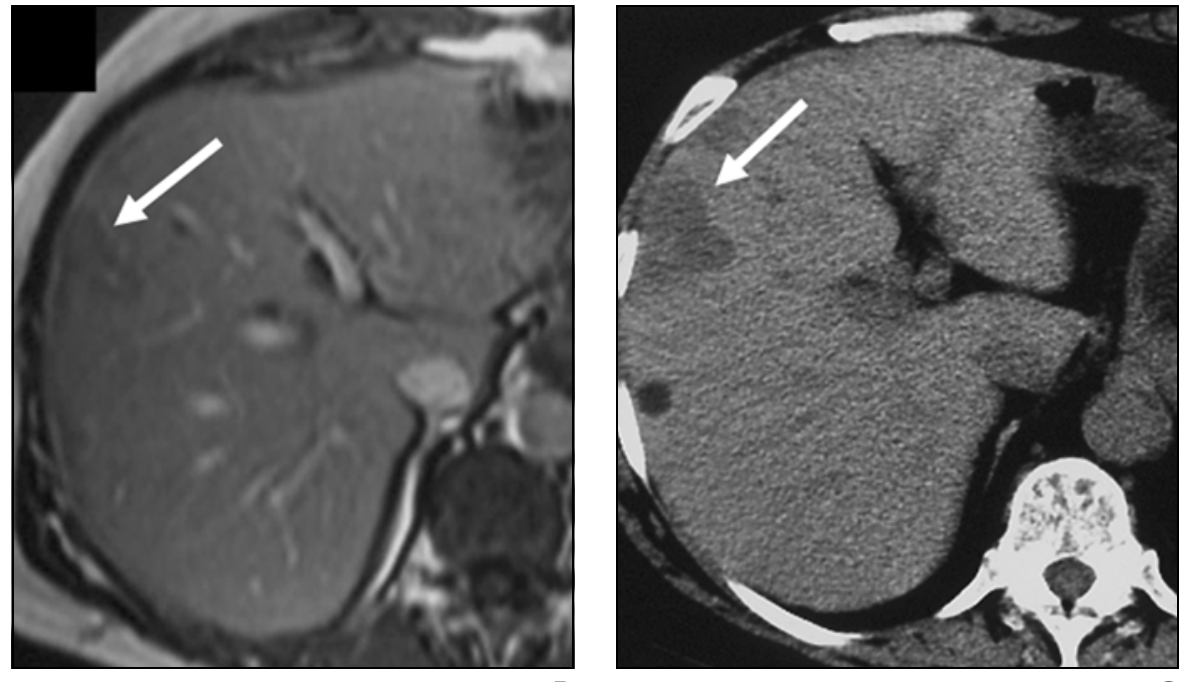

B

C

Fig. 11-65-year-old woman with focal triangular steatosis in liver segment VIII that represents end stage of previous persistent sectorial perfusion alterations caused by hepatic biopsy. Steatosis represents the final outcome of a persistent arterial phenomenon: It may appear to be either irregular (Fig. 10) or regular and sectorial, depending on characteristics of portal vessel damage or injury.

A and B, Axial gradient-echo T1-weighted (TR/TE, 216/1.5) unenhanced (A) and axial T1-weighted (216/1.5) gadolinium-enhanced (B) arterial phase MR images show slightly wedge-shaped hyperintensity compared with surrounding parenchyma and relatively wedge-shaped hypoenhancing area, respectively (arrows).

C, Axial helical CT image confirms triangular hypodense area (arrow) in segment VIII.

D, Axial sonogram (convex, obtained with 3.5-MHz probe) shows sectorial triangular area that is hyperechoic with respect to surrounding liver parenchyma (arrow).

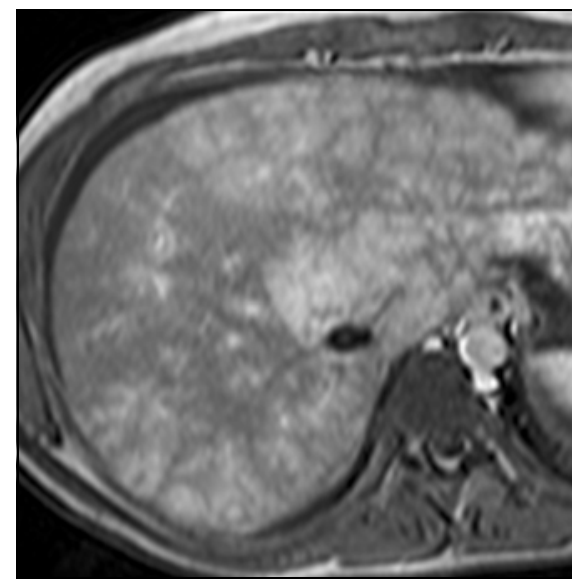

direct trauma, induced inflammation, thrombosis, or arterioportal shunt. These transient hepatic intensity differences may appear either irregular, positioned around or lateral to injury (Fig. 10), or regular and sectorial with regard to the characteristics of portal vessel damage or injury, as in case of a postbiopsy arterioportal shunt (Fig. 3) or portal thrombosis after radiofrequency ablation (Fig. 1). Hemodynamic changes occurring after interventional procedures are often stable, so that a biopsy outcome may be identified as a sectorial area, fatty in normal liver or spare in fatty liver [2, 8] (Fig. 11). 


\section{Transient Hepatic Intensity Differences}

\section{Diffuse Differences}

Transient hepatic signal intensity differences that are diffuse have various patterns depending on the obstacle location and on related compensatory shunts [2, 3]. These intensity differences are secondary to portal hypoperfusion due to blood flow obstruction before, after, or at the level of the sinusoids.
In postsinusoidal obstruction (Budd-Chiari syndrome, right heart failure, and venous occlusive disease), hepatic parenchyma may assume a marbled aspect, defined as a "patchy" pattern (Fig. 12), due to increased IV pressure, consequent transsinusoidal plexus activation, arterial compensation, and general centrolobular enhancement during the arterial phase. In this case, the portal system becomes the only means of drainage for the liver.

When blockade occurs at the level of the portal trunk (before the sinusoids), as in thrombosis, or before the centrolobular vein (into sinusoids), as in cirrhosis, portal flow is adequate for central parenchymal areas but not for the peripheral zones. The arterial

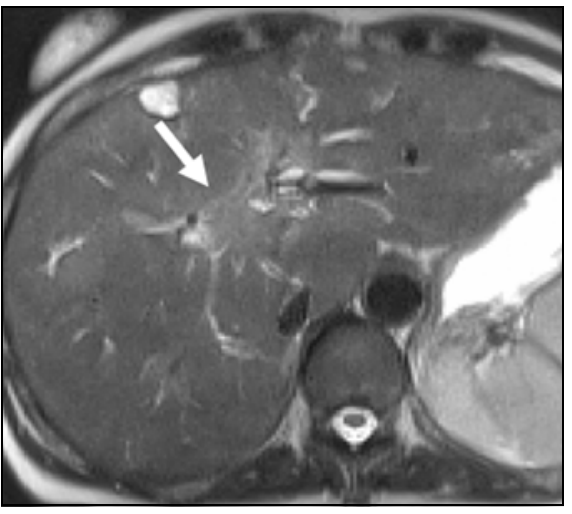

A
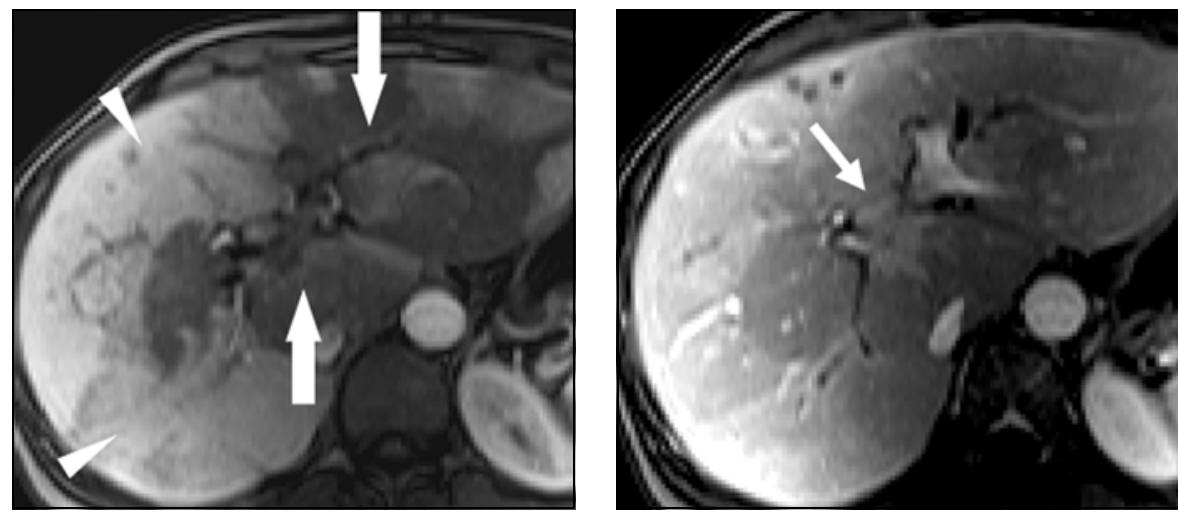

B

C

Fig. 13 - 44-year-old woman with portal trunk and right portal branch obstruction due to central cholangiocellular carcinoma and related diffuse central-peripheral pattern of transient hepatic intensity difference.

A, Axial T2-weighted MR image (TR/TE, 12,000/84) reveals hyperintense mass (arrow) causing portal blood flow obstruction before sinusoids.

B, Axial gradient-echo T1-weighted gadolinium-enhanced arterial phase MR image (146/2) shows diffuse peripheral enhancement of liver parenchyma (arrowheads) and relative hypoperfusion of central areas (arrows).

C, Axial gradient-echo T1-weighted gadolinium-enhanced portal venous phase MR image (146/2) confirms enhanced mass (arrow) and shows central-peripheral pattern is visible but fading (not so evident as on arterial phase image).

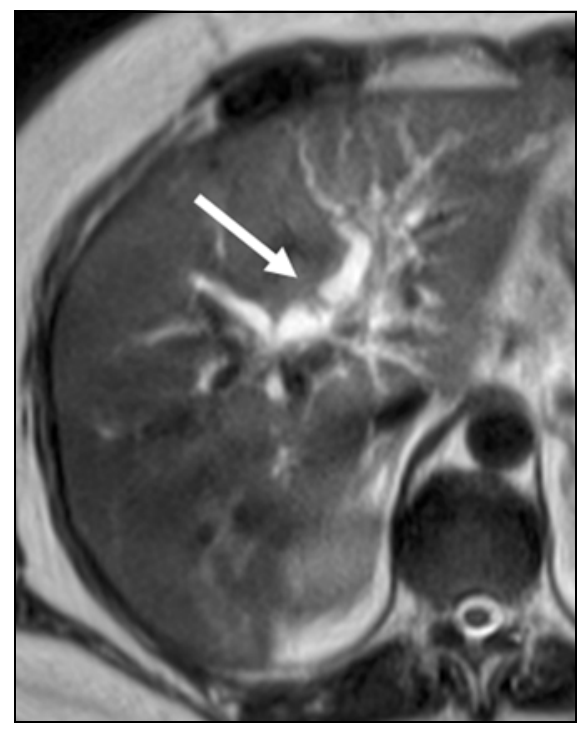

A
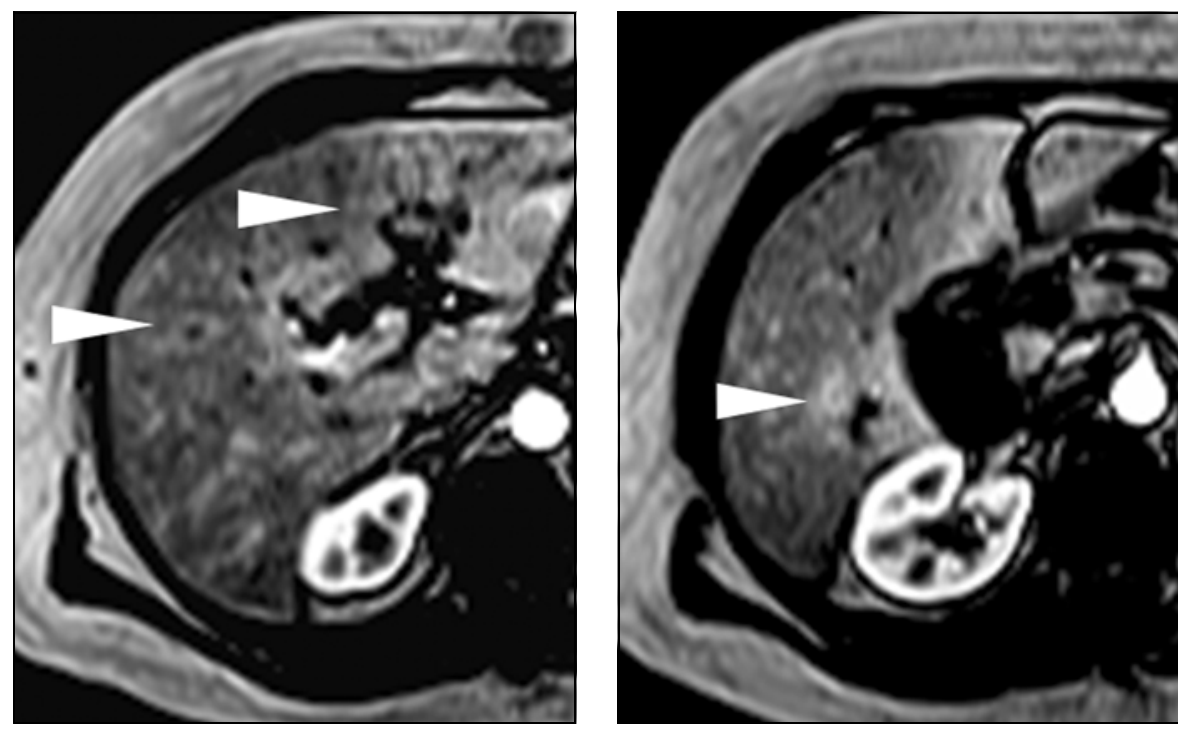

B

C

Fig. 14-51-year-old man with obstruction of main bile duct due to pancreatic head carcinoma (not shown), dilation of entire biliary tree, and diffuse peribiliary pattern of transient hepatic intensity difference.

A, Axial T2-weighted MR image (TR/TE, 12,000/84) shows hyperintense dilated biliary vessels (long-standing biliary obstruction) (arrow) determining impairment of peribiliary plexus.

B and C, Axial gradient-echo T1-weighted gadolinium-enhanced arterial phase MR images (146/2) reveal diffuse cylindric enhancement of liver parenchyma spread along dilated bile ducts (arrowheads). 


\section{Colagrande et al.}

response produces enhancement of subcapsular liver regions with relative hypodensity in the central and perihilar areas. This pattern of enhancement, based on peribiliary plexus activation, is called "central-peripheral" [2, 3] (Fig. 13).

Finally, in biliary tree dilation (choledocholithiasis, cholangiocellular carcinoma, pancreas head carcinoma), the peribiliary plexus may become obstructed, with a consequent decrease in portal blood flow to the sinusoids and arterial compensation. Such long-standing biliary obstruction [2, 3] determines a cylindric arterialization along the dilated biliary vessels, called a "peribiliary" pattern [3] (Fig. 14). Transient hepatic intensity differences associated with biliary disease may have two other appearances: sectorial high intensity, caused by malignant involvement of the portal branches and peribiliary sites, even without any biliary vessel dilation (see Fig. 9 in part 1 [1]); and polymorphous variable-intensity (Figs. 4 and 5) localized arterial phenom- ena proximal to the inflammation site and attributable to edema involving the peribiliary plexus [7].

\section{Conclusion}

Transient hepatic intensity differences are the exact MRI equivalent of transient hepatic attenuation differences on helical CT, so that even on MRI they must be considered important signs of underlying liver disorders, which they help to assess. As a consequence, the hepatic arterial phase must always be performed on MRI as well as on CT, even if no focal lesion is expected to be found.

\section{References}

1. Colagrande S, Centi N, Galdiero R, Ragozzino A. Transient hepatic intensity differences. Part 1, Those associated with focal lesions. AJR 2007; 188:154-159

2. Itai Y, Matsui O. Blood flow and liver imaging. $R a$ diology 1997; 202:306-314

3. Colagrande S, Carmignani L, Pagliari A, Capaccioli
L, Villari N. Transient hepatic attenuation differences (THAD) not connected to focal lesions. $R a$ diol Med 2002; 104:25-43

4. Oliver JH 3rd, Baron RL. Helical biphasic contrastenhanced CT of the liver: technique, indications, interpretation and pitfalls. Radiology 1996; 201:1-14

5. Lane MJ, Jeffrey RB Jr, Katz DS. Spontaneous intrahepatic vascular shunts. AJR 2000; 174:125-131

6. Kim HJ, Kim AY, Kim TK, et al. Transient hepatic attenuation differences in focal hepatic lesions: dynamic CT features. AJR 2005; 184:83-90

7. Arai K, Kawai K, Kohda W, Tatsu H, Matsui O, Nakahama T. Dynamic CT of acute cholangitis: early inhomogeneous enhancement of the liver. AJR 2003; 181:115-118

8. Yoshimitsu K, Honda H, Kuroiwa T, et al. Unusual hemodynamics and pseudolesions of the noncirrhotic liver at CT. RadioGraphics 2001; 21:S81-S96

9. Quiroga S, Sebastia C, Pallisa E, Castella E, PerezLafuente M, Alvarez-Castells A. Improved diagnosis of hepatic perfusion disorders: value of hepatic arterial phase imaging during helical CT. RadioGraphics 2001; 21:65-81

\section{FOR YOUR INFORMATION}

The reader's attention is directed to part I accompanying this article, titled "Transient Hepatic Intensity Differences: Part I, Those Associated with Focal Lesions," which begins on page I54. 